\title{
Elevated expression of serine/threonine phosphatase type 5 correlates with malignant proliferation in human osteosarcoma
}

\author{
Kun Han, Zhihua Gan, Shuchen Lin, Haiyan Hu, Zan Shen and Daliu Min \\ Department of Medical Oncology, The Affiliated 6th People's Hospital of Shanghai Jiaotong University, Shanghai 200223, China
}

\begin{abstract}
Osteosarcoma is the most common primary malignant bone tumor in adolescents and young adults. However, the involvement of serine/threonine phosphatase type 5 (PP5) in osteosarcoma remains unclear. The aim of this study was to evaluate the functional role of PP5 in osteosarcoma cells. Firstly, we found that PP5 is widely expressed in several human osteosarcoma cell lines. Then we used lentivirus-delivered siRNA to silence PP5 expression in Saos-2 and U2OS cell lines. Knockdown of endogenous PP5 expression by shRNA-expressing lentivirus significantly decreased the viability and proliferation of the osteosarcoma cells. Moreover, FACS analysis showed that knockdown of PP5 expression induced a significant arrest in the G0/G1 phase of the cell cycle, which was associated with the inhibition of cell proliferation. Therefore, knockdown of PP5 is likely to provide a novel alternative to targeted therapy of osteosarcoma and deserves further investigation.
\end{abstract}

Key words: cell proliferation, lentivirus, Osteosarcoma, PP5, siRNA, targeted therapy

Received: 04 December, 2014; revised: 22 November, 2015 accepted: 05 May, 2016; available on-line: 13 January, 2017

\footnotetext{
e-mail: daliu_min@163.com

Abbreviations: BCA, bicinchoninic acid; CDNA, complementary DNA; DMEM, Dulbecco's modified Eagle's medium; ECL, enhanced chemiluminescence; FACS, fluorescence-activated cell sorting; FBS, fetal bovine serum; GFP, green fluorescence protein; M-MLV, moloney murine leukemia virus; MOI, multiplicity of infection; MTT, 3-(4, 5-dimethylthiazol-2-yl)-2,5-diphenyl-tetrazolium bromide; $\mathrm{PI}$, propidium iodide; PP5, serine/threonine phosphatase type 5 PVDF, polyvinylidene fluoride; qRT-PCR, quantitative real-time polymerase chain reaction, SDS, sodium dodecyl sulfate; shRNA, short hairpin RNA; siRNA, small interfering RNA; TBST, Tris-buffered saline with Tween 20.
}

\section{INTRODUCTION}

Osteosarcoma is the most common primary bone malignancy in children and young adults ( $\mathrm{Xu}$ et al., 2014), characterized by osteoid producing atypical cells. Its dissemination is typically via the bloodstream, primarily targeting lungs and other bones (Shweikeh et al., 2014). Almost all osteosarcomas are high grade and have a poor prognosis, with $10-20 \%$ of patients having detectable metastases to the lungs at the time of diagnosis. Despite intensive chemotherapy and aggressive surgery, the survival rates of osteosarcoma patients have remained at $50-80 \%$ since the 1970 s (Tian et al., 2014). There is still a significant proportion of osteosarcoma patients with poor response to treatment, who have a high risk of local relapse or distant metastasis (Bacci et al., 2003). In addition, the molecular events which initiate and propa- gate osteosarcoma genesis remain obscure. Thus, the identification of novel and efficient molecular markers is highly necessary for the improvement in treatment of osteosarcoma.

A delicate balance of phosphorylation and dephosphorylation is essential to the maintenance of cellular responsiveness. In the past twenty years, it became evident that serine/threonine phosphatase type 5 (PP5) is a multi-tasking regulator of this balance (Hinds \& Sanchez, 2008). PP5 is a unique member of serine/ threonine phosphatases carrying a regulatory tetratricopeptide repeat (TPR) domain and functions in signaling pathways that control many cellular responses, including DNA damage repair, cell growth and differentiation, cell cycle arrest, and cellular heat shock response (Chinkers, 2001). Previous studies with siRNA and antisense oligonucleotides indicate that a decrease in PP5 expression can result in G1-growth arrest (Zuo et al., 1998; Zuo et al., 1999), DNA-damage checkpoint failure (Zhang et al., 2005), prolonged activation of ASK1 signaling (Morita et al., 2001; Zhou et al., 2004), altered DNA-PKcs signaling (Wechsler et al., 2004), and a GR/p53-dependent increase in p21 expression (Zuo et al., 1998; Zuo et al., 1999).

To date, there have been only a few studies that directly implicate PP5 in the development of cancer. PP5 mRNA levels were reported to be markedly elevated in highly malignant ascites hepatomas in rats (Shirato et al., 2000). Antibody microarray expression studies of mantle-cell lymphomas also indicate PP5 is overexpressed by 2 -folds in cancer samples than in controls (Ghobrial et al., 2005). Golden and coworkers revealed a positive correlation between elevated levels of PP5 and human breast cancer, suggesting that PP5 overexpression may aid tumor progression (Golden et al., 2008a). PP5 gene has been linked to a region of chromosome amplification in osteosarcoma samples from human patients (Atiye et al., 2005). To further evaluate the biological role of PP5 in osteosarcoma, we used a lentivirus-delivered siRNA to silence PP5 expression in human osteosarcoma cell lines.

\section{MATERIALS AND METHODS}

Materials. Dulbecco's modified Eagle's medium (DMEM) was obtained from Hyclone (Logan, Utah, USA; cat no.SH30243.01B+). Fetal bovine serum (FBS) was obtained from Biowest (Loire Valley, France; cat no.S1810). Lipofectamine 2000 and TRIzol® Reagent were purchased from Invitrogen (Carlsbad, CA, USA). M-MLV Reverse Transcriptase was purchased from Promega (Madison, WI, USA). All other chemicals were obtained from Sigma-Aldrich (St. Louis, MO, USA). The lentiviral expression vector $(\mathrm{pFH}-\mathrm{L})$ and packaging vec- 
tors (pVSVG-I and pCMVAR8.92) were purchased from Hollybio (Shanghai, China). The antibodies used were as follows: rabbit anti-PP5 antibody (1:3000 dilution; Abcam, Cambridge, MA, USA; cat no.ab123919), rabbit anti-GAPDH antibody (glyceraldehyde3-phosphate dehydrogenase, 1:100000 dilution; Proteintech Group, Inc., Chicago IL, USA; cat no.10494-1-AP), and horseradish peroxidase-conjugated goat anti-rabbit secondary antibody (1:5000 dilution; Santa Cruz, CA, USA; cat no.SC-2054).

Cell culture. Human osteosarcoma cell lines (Saos-2 and U2OS), osteoblast-like cell line (MG63), chondrosarcoma cell line (SW1353), normal osteoblast cell line (hFOB 1.19) and embryonic kidney cell line (293T) were obtained from the Cell Bank of Chinese Academy of Sciences (Shanghai, China). Human osteosarcoma cell line (SF-86) was from Shanghai Sixth People's Hospital. All cell lines were cultured in DMEM supplemented with $10 \% \mathrm{FBS}$ at $37^{\circ} \mathrm{C}$ with $5 \% \mathrm{CO}_{2}$.

Construction of recombinant lentiviral vector. The cDNA sequence of PP5 was obtained from GenBank (accession number: NM_001204284.1). The shRNA target sequence for PP5 was 5'-GAGACAGAGAAGATTACAGTACTCGAGTACTGTAATCTTCTCTGTCTCT'T'T'T-3', which was subjected to BLAST analysis against the human genome database to eliminate cross-silence phenomena with nontarget genes. A scrambled fragment (5'-GATCCT'TCTCCGAACGTGTCACGTCTCGAGACGACGCACTGGCGGAGAAT'T'T'T'T-3') that has no significant homology with mouse or human gene sequences was used as a negative control. DNA oligonucleotides to produce plasmid-based shRNA were cloned into the pFH-L vector with the use of NheI/PacI restriction sites. The lentiviral expression vector (pFH-L) and packaging vectors (pVSVG-I and pCMV $\Delta$ R8.92) were cotransfected into 293T cells with Lipofectamine 2000 according to the manufacturer's instructions. The supernatant was collected $48 \mathrm{~h}$ later, centrifuged $\left(4000 \times \mathrm{g}, 4^{\circ} \mathrm{C}, 10 \mathrm{~min}\right)$ to remove cell debris, filtered through $0.45-\mu \mathrm{m}$ cellulose acetate filters, and then concentrated again $\left(4000 \times g, 4^{\circ} \mathrm{C}, 15 \mathrm{~min}\right)$. The lentiviral vectors expressed green fluorescence protein (GFP), which allowed for titering and measurement of their infection efficiency in transduced cells. Saos-2 and U2OS cells were dispensed into 6 -well plates at a density of 50000 cells per well and transduced with shRNA-expressing lentivirus at a multiplicity of infection (MOI) of 20 , respectively. GFP expression was observed by fluorescent microscopy four days post-transduction.

Quantitative real-time polymerase chain reaction (qRT-PCR). Cells transduced with shRNA-expressing lentivirus were divided into two groups (Lv-shCon, LvshPP5). Saos-2 and U2OS cells were harvested after lentivirus transduction for four and six days, respectively. Total cellular RNA was extracted using Trizol reagent and reversely transcribed to cDNA by M-MLV reverse transcriptase according to the manufacturer's instructions. ACTB (Actin, Beta) and GAPDH genes were amplified as internal controls. Specific cDNAs were then amplified by qPCR using the following primers: PP5forward, 5'-CCCAACTACTGCGACCAGAT-3'; PP5reverse, 5'-CCCGTCACCTCACATCATTC-3'; ACTBforward, 5'-GTGGACATCCGCAAAGAC-3'; ACTB-reverse, 5'-AAAGGGTGTAACGCAACTA-3'. GAPDHforward, GGAAGCTTGTCATCAATGGAA; GAPDH- reverse, TGGACTCCACGACGTACTCA. qPCR products were detected with SYBR Green on BioRad Connet Real-Time PCR platform. qRT-PCR procedure consisted of: denaturation at $95^{\circ} \mathrm{C}$ for $1 \mathrm{~min}, 40$ cycles of denaturation at $95^{\circ} \mathrm{C}$ for $5 \mathrm{~s}$ and extension at $60^{\circ} \mathrm{C}$ for $20 \mathrm{~s}$. Relative quantitation was analyzed by taking the difference $[\Delta \mathrm{C}(\mathrm{T})]$ between the $\mathrm{C}(\mathrm{T})$ of $\mathrm{ACTB}$ (or GAPDH) and $C(T)$ of PP5 and computing $2[-\Delta \Delta C(\mathrm{~T})]$ as described previously (Sun et al., 2012).

Western blot analysis. Cells transduced with shRNAexpressing lentivirus were divided into two groups (LvshCon, Lv-shPP5). Saos-2 and U2OS cells were harvested after lentivirus transduction for five and seven days, respectively. Total protein was extracted with $2 \times \mathrm{SDS}$ Sample Buffer (100 mM Tris-HCl (pH 6.8), $10 \mathrm{mM}$ EDTA, 4\% SDS, 10\% Glycine). Total protein concentration was determined by the bicinchoninic acid (BCA) assay. Protein extracts were separated by 10\% SDS-PAGE and transferred to PVDF membranes. The membranes were blocked with 5\% nonfat dry milk in Tris-buffered saline with Tween 20 (TBST) for $1 \mathrm{~h}$ at $37^{\circ} \mathrm{C}$, and incubated overnight at $4^{\circ} \mathrm{C}$ in TBST with the anti-PP5 or anti-GAPDH antibody. Following incubation with horseradish peroxidase-conjugated goat anti-rabbit secondary antibody for $1 \mathrm{~h}$, the detection was carried out by using enhanced chemiluminescence (ECL) kit (Amersham) and visualized by exposure to an X-ray film. GAPDH was used as a control to verify equal protein loading.

MTT viability assay. To detect the effect of PP5 inhibition on the reduced number of living cells, 3-(4, 5-dimethylthiazol-2-yl)-2,5-diphenyl-tetrazolium bromide (MT'T) metabolism test was performed four days after lentivirus transduction. Briefly, Saos-2 and U2OS cells were dispensed into 96-well plates at a concentration of 2000 per well. The plates were incubated for one to five days at $37^{\circ} \mathrm{C}$. On each day, MTT $(5 \mathrm{mg} / \mathrm{ml})$ was added and incubated for $4 \mathrm{~h}$. Afterwards, the entire supernatant was discarded and acidic isopropanol $(10 \%$ SDS, $5 \%$ isopropanol and $0.01 \mathrm{~mol} / \mathrm{L} \mathrm{HCl}$ ) was added at a volume of $100 \mu \mathrm{L}$ per well and incubated at $37^{\circ} \mathrm{C}$ for $10 \mathrm{~min}$. The absorbance at $595 \mathrm{~nm}$ of each well was determined using an ELISA reader.

Colony formation assay. To detect the effect of PP5 inhibition on the in vitro tumorigenicity, plate colony formation assay was performed four days after lentivirus transduction. Briefly, Saos-2 and U2OS cells were dispensed into 6-well plates at a concentration of 300 and 200 per well, respectively. The culture medium was changed at regular time intervals. Saos-2 or U2OS cells were cultured for seven or twelve days, until most of single colonies contained more than 50 cells. The colonies were stained with crystal violet for $15 \mathrm{~min}$, and then washed with water and air-dried. Cell colonies were captured and counted under a microscope.

Fluorescence-activated cell sorting analysis (FACS). To detect cell cycle progression, flow cytometry assay was performed seven days after lentivirus transduction as described previously (Gu et al., 2013). Briefly, Saos-2 cells were dispensed into $6-\mathrm{cm}$ dishes at a concentration of 200000 per dish. After culturing at $37^{\circ} \mathrm{C}$ for $40 \mathrm{~h}$, the cells were harvested, fixed in $70 \%$ ethanol, and stored overnight at $4^{\circ} \mathrm{C}$. The cells were then treated with $\mathrm{NaCl} / \mathrm{PI}$ staining solution $(50 \mu \mathrm{g} / \mathrm{mL}$ PI and 100 $\mu \mathrm{g} / \mathrm{mL}$ RNase A). Following incubation for $1 \mathrm{~h}$ in the dark at room temperature, the cells were analyzed by flow cytometry (FACSCalibur; Becton Dickinson, San Jose, CA, USA). Fractions of cells in the G0/G1, S and G2/M phases were analyzed with the dedicated software (Becton Dickinson).

Statistical analysis. Statistical analysis was performed using the SPSS 22.0 software package (SPSS Inc, Chicago, IL, USA). Numerical data are expressed as mean \pm standard deviation. Two means were compared using 
Student's $t$-test. A $P$ value of less than 0.05 was considered statistically significant.

\section{RESULTS}

PP5 is widely expressed in human osteosarcoma cell lines

To explore the role of PP5 in human osteosarcoma, we first detect the expression levels of PP5 in three osteosarcoma cell lines (Saos-2, U2OS and SF-86), one osteoblast-like cell line (MG63), one chondrosarcoma cell line (SW1353) and one osteoblast cell line (hFOB 1.19). As depicted in Fig. 1A, qRT-PCR analysis revealed that PP5 was highly expressed in all osteosarcoma cell lines compared to the normal osteoblast cell line hFOB 1.19. In addition, western blot analysis revealed that the expression levels of PP5 in osteosarcoma cell lines Saos-2 and U2OS, as well as osteoblast-like cell line MG63, were much higher than in normal osteoblast cell line hFOB 1.19 (Fig. 1B). Therefore, we chose Saos-2 and U2OS osteosarcoma cell lines for subsequent investigation of the role of PP5 in osteosarcoma growth.

\section{Knockdown of endogenous PP5 expression by shRNA- expressing lentivirus}

Saos-2 and U2OS cells were transduced with shRNAexpressing lentivirus ( $\mathrm{Lv}$-shCon or $\mathrm{Lv}$-shPP5), respectively. The infection efficiency was confirmed by evaluating GFP expression levels. As depicted in Fig. 2A, over $80 \%$ of cells infected with Lv-shPP5 showed GFP protein expression, which suggests that the lentivirus transduction was successful and highly efficient. The effect of Lv-shPP5 on PP5 expression in Saos- 2 and U2OS cells was observed by qRT-PCR and western blot analysis. As depicted in Fig. 2B to Fig. 2E, the mRNA and protein expression levels of PP5 were weaker in the cells infected with Lv-shPP5 than in cells infected with Lv-shCon. It shows that after Lv-shPP5 infection, the transcription
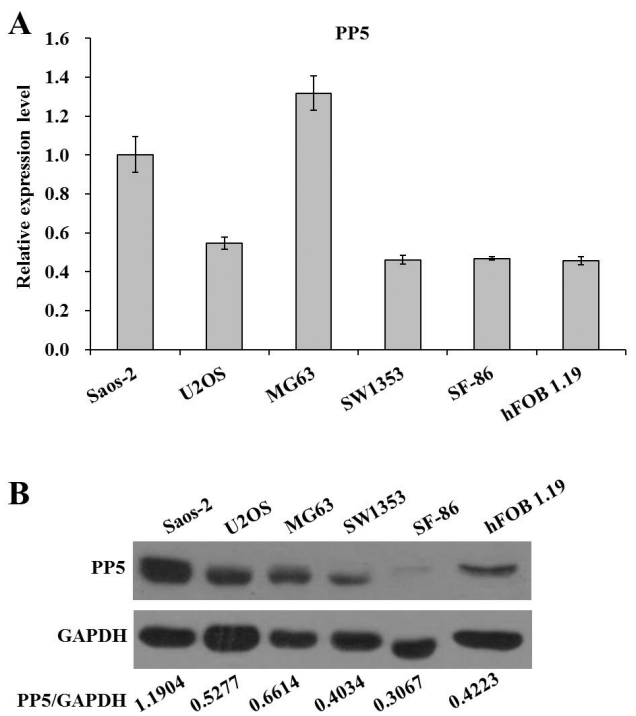

Figure 1. Expression levels of PP5 in human osteosarcoma cell lines.

(A) Quantitative analysis of PP5 mRNA expression in Saos-2, U2OS, MG63, SW1353, SF-86 cell, and HFOB 1.19 lines assessed by qRTPCR. $\beta$-actin and GAPDH genes were used as the internal controls. (B) Representative western blot showing PP5 expression determined in all these six cell lines. GAPDH protein was used as an internal control. Data are mean \pm S.D. ( $n=3$; $t$-test).

level was reduced by about $80 \%$ in both Saos- 2 and U2OS cells. Therefore, Lv-shPP5 could specifically and strongly abolish endogenous PP5 expression in osteosarcoma cells.

\section{Knockdown of PP5 expression disrupts cell proliferation}

After infection by PP5 shRNA-expressing lentivirus for four days, we investigated the cell viability for five consecu-
A Saos-2 cells

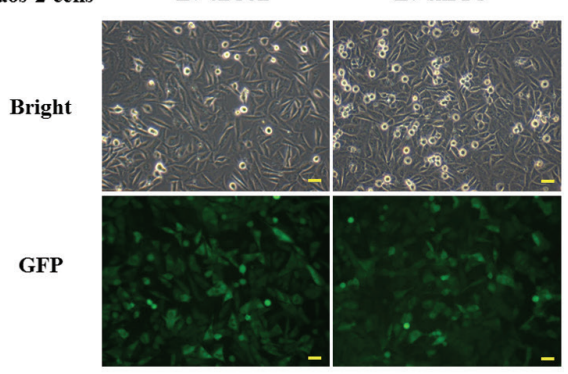

U2OS cells

Bright
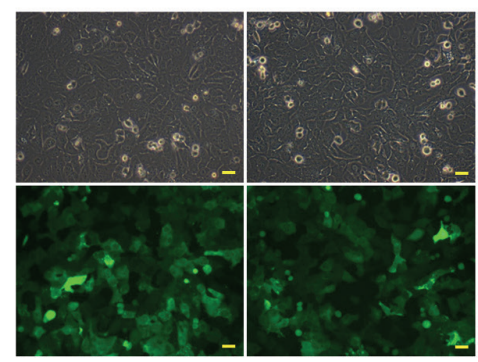

B

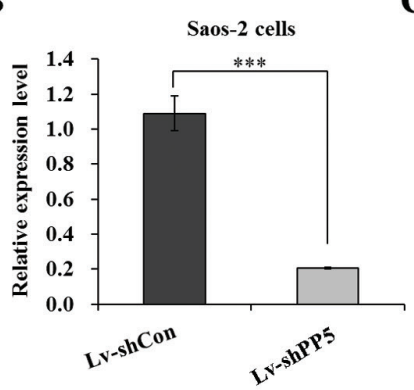

D
C

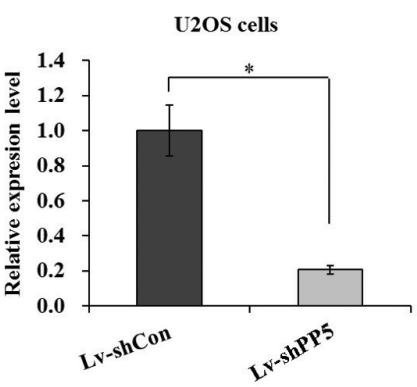

$\mathbf{E}$

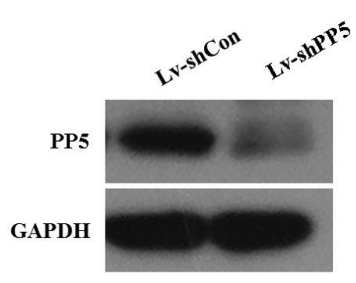

Figure 2. Lentivirus stably expressing shRNA targeting PP5 is successfully constructed.

(A) Evaluation of the lentivirus transduction rate, which was more than $80 \%$ as calculated by cellular enumeration using fluorescence and light microscopy. (B, C) Quantitative analysis of PP5 knockdown efficiency in Saos-2 and U2OS cells assessed by qRT-PCR. $\beta$-actin and GAPDH genes were used as the internal controls. (D, E) Representative western blot showing PP5 knockdown efficiency determined in Saos-2 and U2OS cells. GAPDH protein was used as an internal control. Data are mean \pm S.D. ( $\mathrm{n}=3$; $t$-test). ${ }^{*} P<0.05 ;{ }^{* * *} P<0.001 ;$ scale bar, $100 \mu \mathrm{m}$. 
A

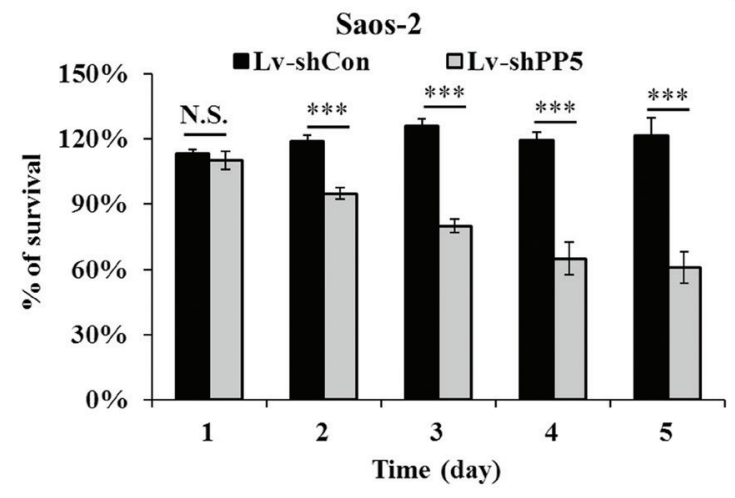

B

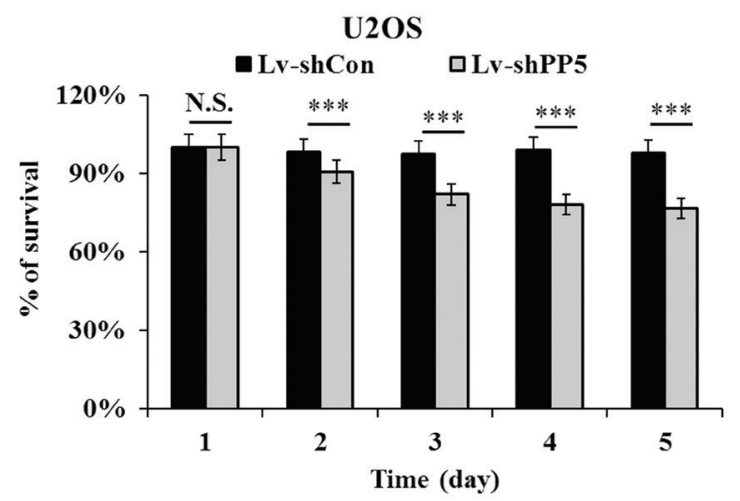

Figure 3. Depletion of PP5 inhibits osteosarcoma cell proliferation.

Cell proliferation was measured using the MTT assay at $\mathrm{OD}_{595 \mathrm{~nm}}$ in Saos-2 (A) and U2OS cells (B). The number of viable cells was fewer in case of cells infected with Lv-shPP5 than in case of cells infected with Lv-shCon. Data are mean \pm S.D. ( $n=3 ; t$-test). ${ }^{* *} P<0.001$. N.S. indicates the difference is not significant.

tive days by the MTT assay. As depicted in Fig. 3A and 3B, the proportion of living cells infected with Lv-shPP5 was significantly reduced $(P<0.001)$ when compared to those infected with Lv-shCon. This indicates that Lv-shPP5 could strongly decrease the viability of osteosarcoma cells.

In addition, colony formation assay was performed to determine cell proliferation in vitro. Consistently, the colony formation was significantly disrupted $(P<0.05)$ in the cells infected with Lv-shPP5. Representative graphs are presented in Fig. 4A and 4B. Crystal violet staining and fluorescence expression showed that both, the size of the mon-

A
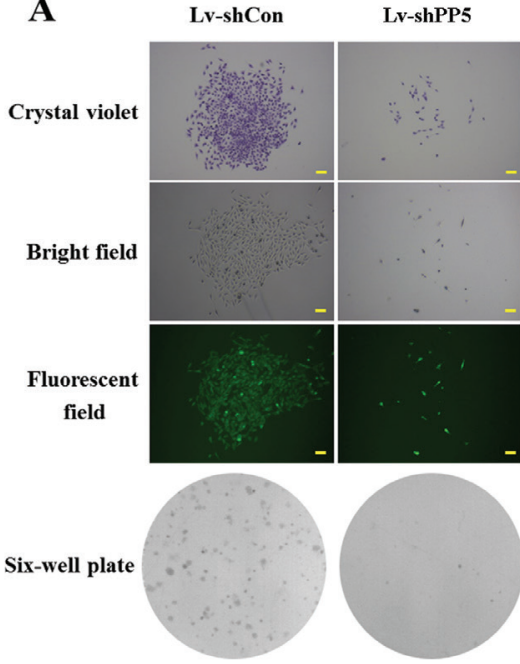

C

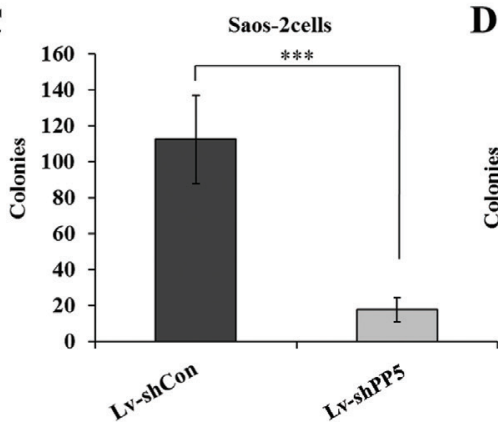

oclone and the number of total colonies, were decreased in cells infected with Lv-shPP5. The number of colonies formed by Saos- 2 and U2OS cells was decreased by $83.9 \%$ and $70.0 \%$, respectively, in comparison with the control (Fig. 4C and 4D). This indicates that Lv-shPP5 could efficiently disrupt the colony formation of osteosarcoma cells.

\section{Knockdown of PP5 expression affects cell cycle}

Flow cytometry assay was used to determine the effect of Lv-shPP5 on the cell cycle of U2OS cells. Rep-
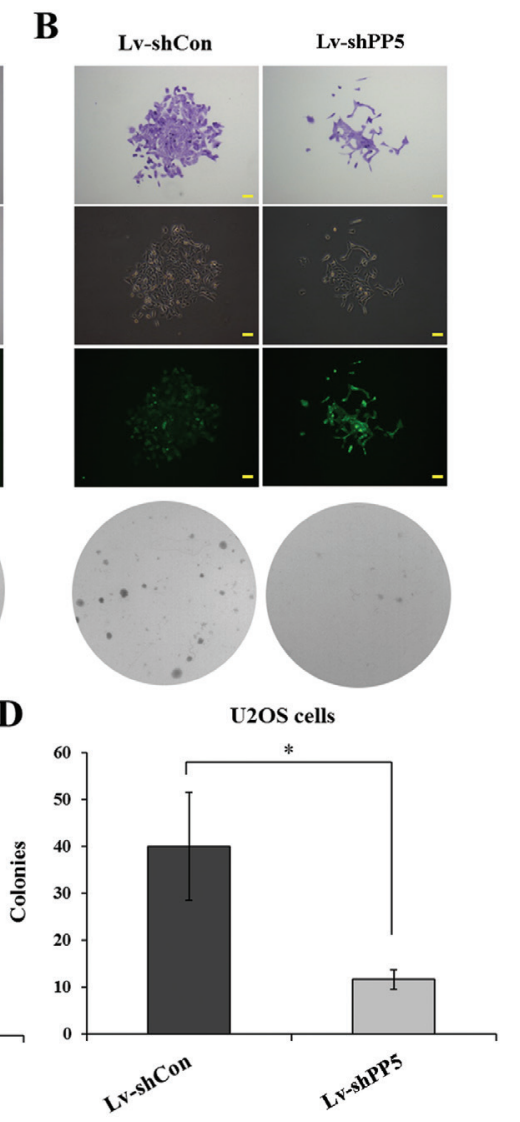

Figure 4. Depletion of PP5 inhibits osteosarcoma cell colony formation.

(A, B) Representative colony formation showing clonogenic survival determined in Saos-2 and U2OS cells. (C, D) The number of colonies was fewer in cells infected with Lv-shPP5 than in cells infected with Lv-shCon. Data are mean \pm S.D. $\left(n=3 ; t\right.$-test). ${ }^{*} P<0.05 ;{ }^{* * *} P<0.001$; scale bar, $250 \mu \mathrm{m}$. 


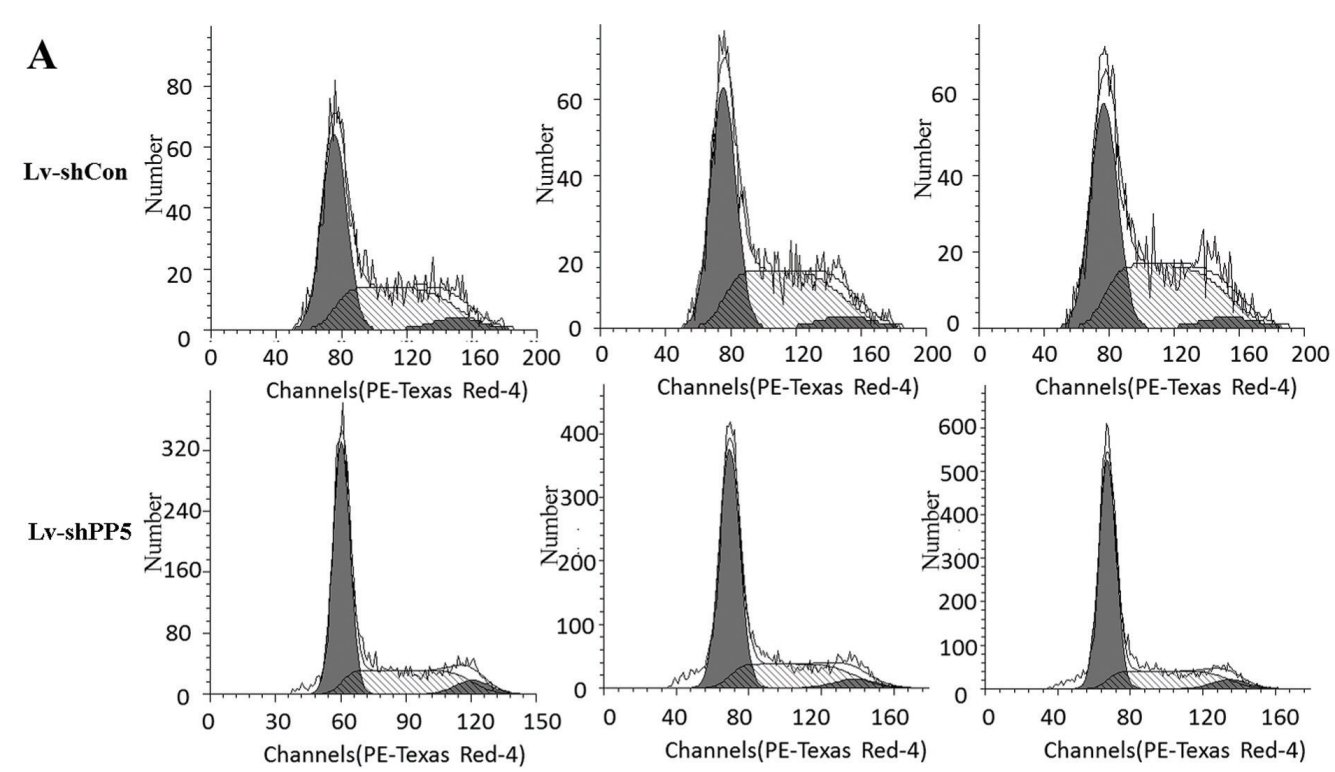

B

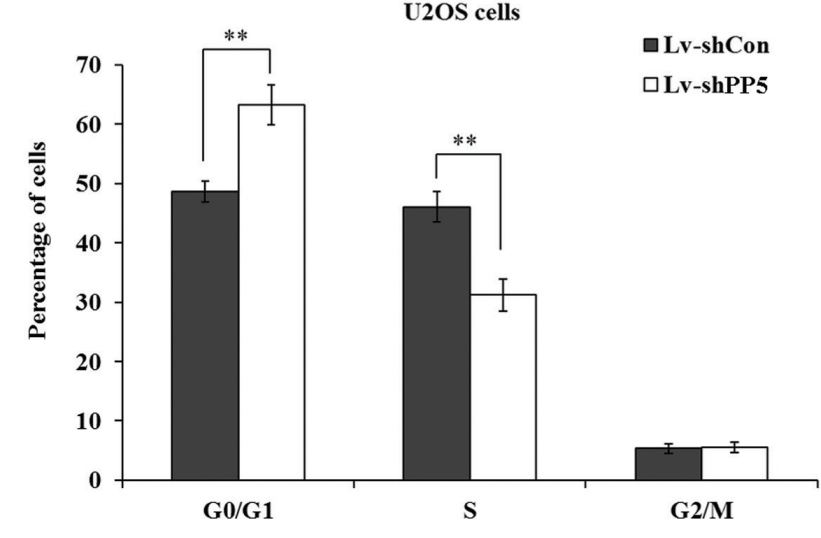

Figure 5. Depletion of PP5 arrests cell cycle progression in U2OS cells.

(A) Comparison of the cell population in the G0/G1, S and G2/M phase between the Lv-shCon and Lv-shPP5 groups as assessed by flow cytometry. (B) The percentage of cells in the G0/G1 phase was significantly higher in the cells infected with LV-shPP5 than in the cells infected with Lv-shCon, while the percentages of cells in the $S$ phase were simultaneously reduced. Data are mean \pm S.D. ( $n=3 ; t$-test). $* * 0<01$.

resentative images of cell cycle distribution are presented in Fig. 5A. As depicted in Fig. 5B, the cell percentage in the G0/G1 phase was increased from $48.61 \%$ in cells infected with Lv-shCon, to $63.29 \%$ in cells infected with Lv-shPP5. Meanwhile, the cell percentage in the S phase was decreased from $46.06 \%$ in cells infected with LvshCon, to $31.21 \%$ in cells infected with Lv-shPP5. This indicates that Lv-shPP5 could strongly block $(P<0.01)$ the cell cycle progression of osteosarcoma cells. Taken together, these results suggest knockdown of PP5 could inhibit osteosarcoma cell proliferation by inducing the G0/G1 phase cell cycle arrest.

\section{DISCUSSION}

Although the aberrant actions of protein kinases have been long known to contribute to tumor promotion and carcinogenesis, the roles for protein phosphatases in the development of human cancer have only emerged in the last decade (Golden et al., 2008b). Although the PP5 gene has been linked to a region of chromosome amplification in patients with osteosarcoma, there is no direct experimental proof that PP5 overexpression aids in osteosarcoma promotion or carcinogenesis.
In this study, we provide substantial data demonstrating that PP5 potentially plays a key role in the development of osteosarcoma. Knockdown of endogenous PP5 expression by shRNA-expressing lentivirus significantly decreased the viability and proliferation of the osteosarcoma cells. Moreover, FACS analysis showed that knockdown of PP5 expression induced a significant arrest in the G0/G1 phase of cell cycle, which was helpful in the inhibition of cell proliferation. Our results are supported by previous reports that suppression of PP5 expression with siRNA and antisense oligonucleotides result in the G1-growth arrest (Zuo et al., 1998; Zuo et al., 1999). Therefore, it seems likely that aberrant PP5 expression may indeed contribute to a neoplastic transformation and cancer progression.

It has been reported that PP5 could affect common components which are involved in expression of a variety of tumor related molecules as p53, ASK-1, ATR, DNA-PKs. Recognition of the biological function of PP5 should not only provide a new insight into cell behavior mechanisms, but also facilitate the development of targeted human cancer therapies (Golden et al., 2008b). 
In summary, our findings provide substantial data demonstrating that PP5 plays an essential role in osteosarcoma growth and may be a potent therapeutic target in human osteosarcoma. However, further investigation is strongly needed to clarify the molecular basis for the oncogenic function of PP5 in osteosarcoma cells.

\section{Acknowledgement}

The authors are thankful for the financial support from the National Nature Science Foundation of China (No. 81172548 and No. 81172105).

\section{Conflict of interests}

The authors declare no conflict of interests.

\section{REFERENCES}

Atiye J, Wolf M, Kaur S, Monni O, Bohling T, Kivioja A, Tas E, Serra M, Tarkkanen M, Knuutila S (2005) Gene amplifications in osteosarcoma-CGH microarray analysis. Genes Chromosomes Cancer 42: 158-163. doi: 10.1002/gcc.20120

Bacci G, Briccoli A, Rocca M, Ferrari S, Donati D, Longhi A, Bertoni F, Bacchini P, Giacomini S, Forni C, Manfrini M, Galletti S (2003) Neoadjuvant chemotherapy for osteosarcoma of the extremities with metastases at presentation: recent experience at the Rizzoli Institute in 57 patients treated with cisplatin, doxorubicin, and a high dose of methotrexate and ifosfamide. Ann Oncol 14: 1126-1134.

Chinkers M (2001) Protein phosphatase 5 in signal transduction. Trends Endocrinol Metab 12: 28-32

Ghobrial IM, McCormick DJ, Kaufmann SH, Leontovich AA, Loegering DA, Dai NT, Krajnik KL, Stenson MJ, Melhem MF, Novak AJ, Ansell SM, Witzig TE (2005) Proteomic analysis of mantle-cell lymphoma by protein microarray. Blood 105: 3722-3730. doi: 10.1182/blood-2004-10-3999

Golden T, Aragon IV, Rutland B, Tucker JA, Shevde LA, Samant RS, Zhou G, Amable L, Skarra D, Honkanen RE (2008a) Elevated levels of Ser/Thr protein phosphatase 5 (PP5) in human breast cancer. Biochim Biophys Acta 1782: 259-270. doi: 10.1016/j.bbadis. 2008.01 .004

Golden T, Swingle M, Honkanen RE (2008b) The role of serine/ threonine protein phosphatase type 5 (PP5) in the regulation of stress-induced signaling networks and cancer. Cancer Metastasis Rev 27: 169-178. doi: $10.1007 /$ s10555-008-9125-z
Gu J, Li K, Li M, Wu X, Zhang L, Ding Q, Wu W, Yang J, Mu J, Wen H, Ding Q, Lu J, Hao Y, Chen L, Zhang W, Li S, Liu Y (2013) A role for p21-activated kinase 7 in the development of gastric cancer. FEBS J 280: 46-55. doi: 10.1111/febs.12048

Hinds TD, Jr., Sanchez ER (2008) Protein phosphatase 5. Int J Biochem Cell Biol 40: 2358-2362. doi: 10.1016/j.biocel.2007.08.010

Morita K, Saitoh M, Tobiume K, Matsuura H, Enomoto S, Nishitoh H, Ichijo H (2001) Negative feedback regulation of ASK1 by protein phosphatase 5 (PP5) in response to oxidative stress. EMBO J 20: 6028-6036. doi: 10.1093/emboj/20.21.6028

Shirato H, Shima H, Nakagama H, Fukuda H, Watanabe Y, Ogawa K, Matsuda Y, Kikuchi K (2000) Expression in hepatomas and chromosomal localization of rat protein phosphatase 5 gene. Int J Oncol 17: 909-912.

Shweikeh F, Bukavina L, Saeed K, Sarkis R, Suneja A, Sweiss F, Drazin D (2014) Brain metastasis in bone and soft tissue cancers: a review of incidence, interventions, and outcomes. Sarcoma 2014: 475175. doi: 10.1155/2014/475175

Sun W, Yao L, Jiang B, Shao H, Zhao Y, Wang Q (2012) A role for Cdkl1 in the development of gastric cancer. Acta Oncol 51: 790-796. doi: 10.3109/0284186X.2012.665611

Tian J, Li X, Si M, Liu T, Li J (2014) CD271+ Osteosarcoma Cells Display Stem-Like Properties. PLoS One 9: e98549. doi: 10.1371/ journal.pone.0098549

Wechsler T, Chen BP, Harper R, Morotomi-Yano K, Huang BC, Meek K, Cleaver JE, Chen DJ, Wabl M (2004) DNA-PKcs function regulated specifically by protein phosphatase 5. Proc Natl Acad Sci USA 101: 1247-1252. doi: 10.1073 / pnas.0307765100

Xu H, Liu X, Zhao J (2014) Down-regulation of miR-3928 promoted osteosarcoma growth. Cell Physiol Biochem 33: 1547-1556. doi: 10.1159/000358718

Zhang J, Bao S, Furumai R, Kucera KS, Ali A, Dean NM, Wang $\mathrm{XF}$ (2005) Protein phosphatase 5 is required for ATR-mediated checkpoint activation. Mol Cell Biol 25: 9910-9919. doi: 10.1128/ MCB.25.22.9910-9919.2005

Zhou G, Golden T, Aragon IV, Honkanen RE (2004) Ser/Thr protein phosphatase 5 inactivates hypoxia-induced activation of an apoptosis signal-regulating kinase $1 / \mathrm{MKK}-4 / \mathrm{JNK}$ signaling cascade. J Biol Chem 279: 46595-46605. doi: 10.1074/ibc.M408320200

Zuo Z, Dean NM, Honkanen RE (1998) Serine/threonine protein phosphatase type 5 acts upstream of p53 to regulate the induction of p21(WAF1/Cip1) and mediate growth arrest. I Biol Chem 273: $12250-12258$

Zuo Z, Urban G, Scammell JG, Dean NM, McLean TK, Aragon I, Honkanen RE (1999) Ser/Thr protein phosphatase type 5 (PP5) is a negative regulator of glucocorticoid receptor-mediated growth arrest. Biochemistry 38: 8849-8857. doi: 10.1021/bi990842e 